\title{
Hypoxia-inducible gene 2 promotes the immune escape of hepatocellular carcinoma from nature killer cells through the interleukin-10-STAT3 signaling pathway
}

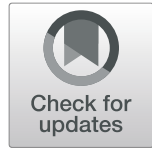

Chuanbao Cui ${ }^{1}$, Kaiwen Fu' ${ }^{2}$, Lu Yang ${ }^{1}$, Shuzhi Wu${ }^{1}$, Zuojie Cen ${ }^{1}$, Xingxing Meng ${ }^{1}$, Qiongguang Huang ${ }^{1}$ and Zhichun Xie ${ }^{1 *}$

\begin{abstract}
Background: The study examines the expression and function of hypoxia-inducible gene 2 (HIG2) in hepatocellular carcinoma (HCC) tissues and cells.

Methods: Forty patients with HCC were included in the study. Bioinformatic analysis was used to analyze the clinical relevance of HIG2 expression in HCC tissue samples. Immunohistochemistry was employed to determine the expression of target proteins in tumor tissues. Hepatic HepG2 and SMMC-7721 cells were transfected with HIG2targeting siRNA with Lipofectamine 2000. qRT-PCR was carried out to determine gene expression levels, while Western blotting was used to determine protein expression. A CCK-8 assay was performed to detect proliferation of cells, while migration and invasion of cells were studied by Transwell assay. Flow cytometry was carried out to detect surface markers and effector molecules in Nature killercells, as well as the killing effect of NK cells.

Results: HIG2 expression was upregulated in HCC. Silencing of HIG2 suppressed HCC cell migration and invasion. The killing effect of NK cells on HCC cells was enhanced after HIG2 was silenced in HCC cells. Conditioned media from HIG2-silenced SMMC-7721 cells inhibited the phenotype and function of NK cells. HCC cells with silenced expression of HIG2 modulated the activity of NK cells via STAT3. HIG2 promoted the evasion of HCC cells from killing by NK cells through upregulation of IL-10 expression.

Conclusion: The study demonstrates that HIG2 activates the STAT3 signaling pathway in NK cells by promoting IL10 release by HCC cells, thereby inhibiting the killing activity of NK cells, and subsequently promoting the recurrence and metastasis of HCC.
\end{abstract}

Keywords: Hypoxia-inducible gene 2, Hepatocellular carcinoma, Nature killer cells, IL-10, STAT3

\section{Background}

Hepatocellular carcinoma (HCC) is one of the most common malignant tumors in the world, and its incidence is higher in men than in women $[1,2]$. In China, the incidence of HCC ranks fourth among all malignant tumors, and its mortality rate ranks second [3]. At present, surgical resection is still the first choice for treating $\mathrm{HCC}$, but the

\footnotetext{
* Correspondence: 357154887@qq.com

'Department of Epidemiology, Guangxi Medical University, No. 22

Shuangyong Road, Nanning 530021, Guangxi Zhuang Autonomous Region,

People's Republic of China

Full list of author information is available at the end of the article
}

prognosis is poor after radical surgery, with a 5-year survival rate of approximately $16 \%$ [4]. Recurrence and metastasis of HCC are key factors that limit clinical outcomes. It has been reported that the recurrence and metastasis of HCC are complex processes, which mainly include inactivation or mutation of tumor-suppressor genes and abnormal activation of oncogenes [5, 6]. The molecular mechanism of recurrence and metastasis of HCC remains unclear. Therefore, studying the mechanism of $\mathrm{HCC}$ at the molecular level and finding effective therapeutic measures have become of great scientific and clinical importance in HCC. 
Hypoxia-inducible gene 2 (HIG2), which is located at q32.1 of human chromosome 7 , is a newly discovered gene that can be induced by hypoxia and lack of glucose. With a complete length of $3.4 \mathrm{~kb}$, it contains two exons and one intron [7, 8]. Expression of HIG2 is induced in hypoxic environments, and HIG2 has been proven to be a target gene of hypoxia-inducible factor-1 (HIF-1) [9]. It has been reported that HIG2 is a new type of lipid droplet (LD) protein, which stimulates the accumulation of lipids in cells [10]. In recent years, the role of the HIG2 gene in the occurrence and development of tumors has garnered significant research interest. Studies have shown that HIG2 plays an important role in the development and progression of renal cell carcinoma, cell lymphoma, epithelial ovarian cancer, transparent cell adenocarcinoma, and uterine cancer $[11,12]$.

Innate immunity is the first line of defense against microbial infection and cancers [13]. Natural killer cells are the most important natural immune cells, and have powerful tumor-killing functions. Natural killer (NK) cells are derived from the bone marrow, and account for $10-18 \%$ of peripheral blood mononuclear lymphocytes [14]. NK cells can be phenotyped as $\mathrm{CD}^{-} \mathrm{CD}^{+} 6^{+}$lymphocytes. Animal and clinical experiments have confirmed that the number and activity of NK cells are directly related to tumorigenesis and prognosis [15]. Higher number and activity of NK cells usually correspond to stronger suppression of tumors. Tumor tissues are infiltrated by a large number of NK cells, and tumor cells with high metastatic potential need to escape immune surveillance before metastasis can occur [5]. However, the activity and function of NK cells that infiltrate tumor tissues are inhibited in varying degrees. If the inhibition of NK cells by the tumor microenvironment can be relieved, the killing effect of NK cells on tumors can be restored [16]. As the main component of tumors, tumor cells can have a strong regulatory effect on the tumor microenvironment [17]. However, this underlying mechanism still needs to be further explored. In the present study, we examine the expression and function of HIG2 in HCC tissues and cells and investigate the effect of HIG2 on HCC cell regulation of the immunological function of NK cells.

\section{Materials and methods \\ Patients}

A total of 40 patients with $\mathrm{HCC}$ who underwent surgical resection at Chongqing Cancer Hospital between January 2016 and December 2017 were included in the study (29 males and 11 females; age range, $32-55$ years; mean age, 43.6 years). None of the patients had a history of any other types of malignant tumors or chemoradiotherapy. Among the patients, 22 cases had lymph node metastasis and 18 cases had no lymph node metastasis.
According to the 2003 TNM staging standards by the Union for International Cancer Control, 11 cases were Stage I, 16 cases were Stage II, 5 cases were Stage III, and 8 cases were Stage IV. HCC tissues and tumoradjacent tissues were resected from all patients and included in the experimental and control groups, respectively. All procedures performed in the current study were approved by the Ethics Committee of Chongqing Cancer Hospital. A written informed consent was obtained from all patients or their families.

\section{Bioinformatics}

Bioinformatic analysis was used to analyze the clinical relevance of HIG2 gene expression in HCC tissues. We utilized the Gene Expression Profiling Interactive Analysis (GEPIA) database (http://gepia.cancer-pku.cn/) to assess the correlation between HIG2 expression and 5year overall survival, and disease-free survival of HCC patients.

\section{Immunohistochemistry}

Freshly resected liver tissues were fixed overnight with $4 \%$ paraformaldehyde and paraffin-embedded before being sectioned at $4 \mu \mathrm{m}$. Paraffin sections were dewaxed at $67^{\circ} \mathrm{C}$ for $2 \mathrm{~h}$ before being washed three times with phosphate-buffered saline (PBS) for $3 \mathrm{~min}$ each time. Dewaxed tissue slices were boiled for $20 \mathrm{~min}$ in citrate buffer $(\mathrm{pH}=6.0)$ and cooled to room temperature. After washing with PBS twice, slides were each covered with $3 \% \mathrm{H}_{2} \mathrm{O}_{2}$ and then incubated at $37^{\circ} \mathrm{C}$ for $10 \mathrm{~min}$. After washing with PBS, slides were each covered with $100 \mu \mathrm{l}$ of HIG2 and IL-10 primary antibodies (1:50 dilution for both) and incubated at room temperature for $2 \mathrm{~h}$. After washing with PBS, slides were each covered with $100 \mu \mathrm{l}$ of polymer enhancer before incubation at room temperature for $20 \mathrm{~min}$. After washing with PBS, slides were each covered with $100 \mu \mathrm{l}$ of enzyme-labeled antimouse / rabbit polymers before incubation at room temperature for $1 \mathrm{~h}$. After washing with PBS, slides were each covered with 1 drop of diaminobenzidine (DAB) and observed under a microscope after $5 \mathrm{~min}$. Slides were then stained with hematoxylin, differentiated with $0.1 \% \mathrm{HCl}$, and washed with water. The slides were then dehydrated using an increasing alcohol gradient, vitrificated by dimethylbenzene, and fixed by neutral balata. After drying, the slice was observed under a light microscope.

\section{Cells}

Hepatic HepG2 and SMMC-7721 cells (Cell Bank, Chinese Academy of Sciences, Shanghai, China) were cultured in DMEM supplemented with $10 \%$ fetal bovine serum (FBS), $100 \mathrm{IU} / \mathrm{ml}$ penicillin, and $100 \mathrm{IU} / \mathrm{ml}$ streptomycin (all reagents from Thermo Fisher 


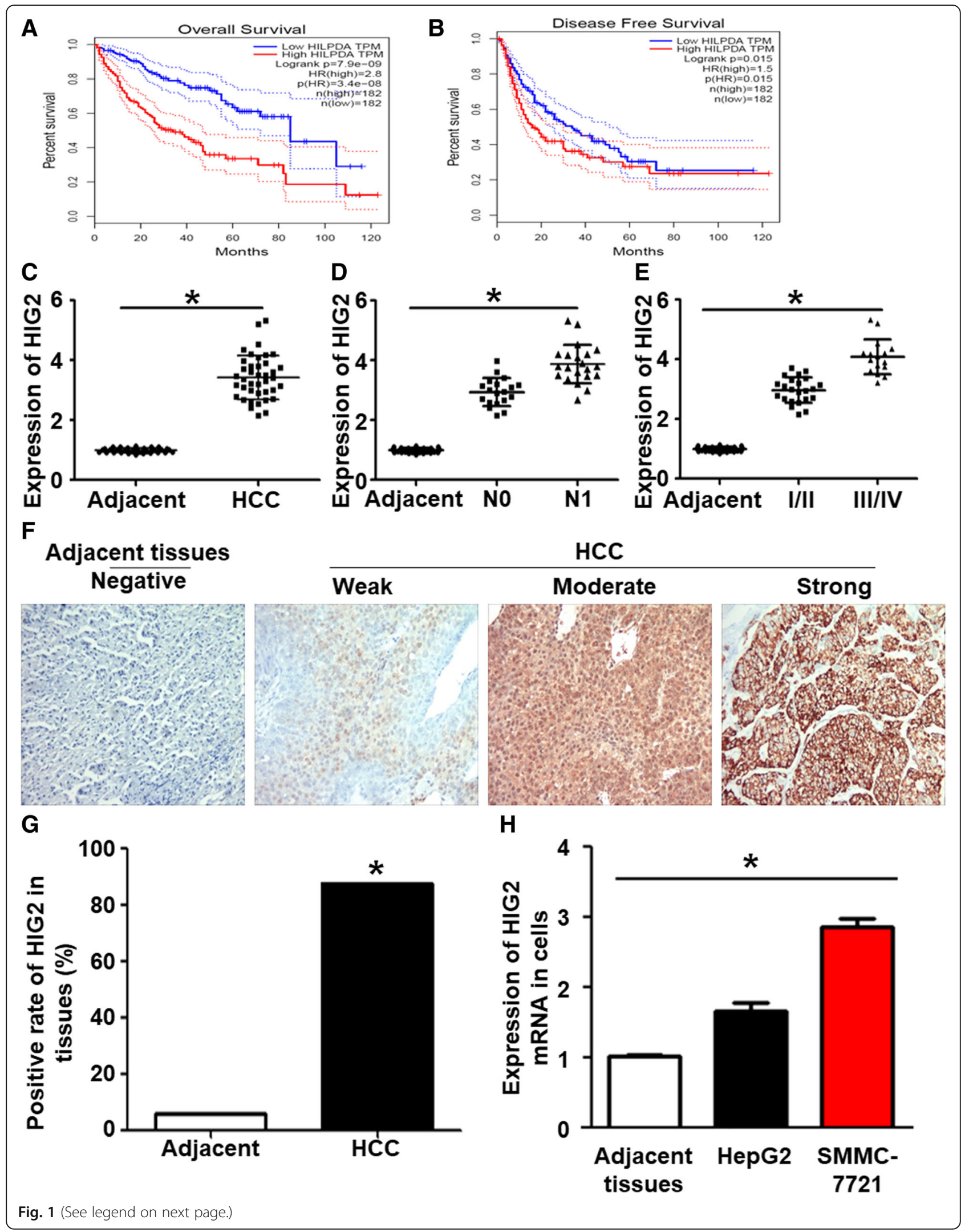


(See figure on previous page.)

Fig. 1 Expression of HIG2 in HCC tissues and cells. a Overall survival of HCC patients with different levels of HIG2 expression. b Disease-free survival of HCC patients with different levels of HIG2 expression. c HIG2 mRNA expression in HCC tissues in comparison with tumor-adjacent tissues. ${ }^{*} P<0.05$. $\mathbf{d}$ HIG2 mRNA expression in HCC tissues from patients with or without lymph node metastasis in comparison to tumor-adjacent tissues. ${ }^{*} P<0.05$. e HIG2 mRNA expression in HCC tissues from patients with TNM Stage I/II or III/IV disease in comparison to tumor-adjacent tissues. ${ }^{*} P<0.05$. $\mathbf{f}$ Immunohistochemical analysis of HIG2 expression in HCC tissues and tumor-adjacent tissues. $\mathbf{g}$ High incidence of HIG2 expression in HCC tissues in comparison to tumor-adjacent tissues. ${ }^{*} P<0.05$. $\mathbf{h}$ Expression of HIG2 mRNA in HepG2 and SMMC-7721 cells in comparison to tumor-adjacent tissues. ${ }^{*} P<0.05$

Scientific, Waltham, MA, USA) at $37^{\circ} \mathrm{C}, 5 \% \mathrm{CO}_{2}$ and $70 \%$ humidity. The cells were passaged every 3 days, and those in logarithmic growth were collected for further assays.

One day before transfection, HepG2 and SMMC-7721 cells $\left(2 \times 10^{5}\right)$ in logarithmic growth were seeded in 24well plates containing antibiotics-free DMEM supplemented with $10 \%$ FBS. Cells were transfected at $70 \%$ confluence. In the first vial, $1.5 \mu \mathrm{L}$ siR-NC or siR-HIG2 (20 pmol $/ \mu \mathrm{L}$; Hanbio Biotechnology Co., Ltd., Shanghai, China) was mixed with $50 \mu \mathrm{l}$ Opti Mem medium (Thermo Fisher Scientific). In the second vial, $1 \mu \mathrm{L}$ Lipofectamine 2000 (Thermo Fisher Scientific) was mixed with $50 \mu \mathrm{l}$ Opti Mem medium. After a 5-min incubation, the two vials were combined and the mixture was incubated at room temperature for $20 \mathrm{~min}$. The mixtures were then added onto cells in the respective groups. Six hours later, the medium was replaced with DMEM containing 10\% FBS. After cultivation for $48 \mathrm{~h}$, the cells were collected for further assays.

To isolate peripheral blood mononuclear lymphocytes, $3 \mathrm{ml}$ peripheral blood was gently added onto the surface of $3 \mathrm{ml}$ Ficoll solution (MagniSort ${ }^{\mathrm{TM}}$ Mouse NK cell Enrichment Kit; Thermo Fisher Scientific, Waltham, MA, USA), followed by centrifugation at $650 \times \mathrm{g}$ and $4{ }^{\circ} \mathrm{C}$ for $20 \mathrm{~min}$. The middle layer containing lymphocytes was carefully transferred to a new $15 \mathrm{ml}$ tube. The separated lymphocytes were mixed with PBS up to a maximum volume of $10 \mathrm{ml}$ and centrifuged at $250 \mathrm{x}$ g and $4{ }^{\circ} \mathrm{C}$ for $10 \mathrm{~min}$. The cell pellet was resuspended in $5 \mathrm{ml}$ PBS and then centrifuged at $250 \mathrm{x}$ g for $10 \mathrm{~min}$. Isolated peripheral blood mononuclear lymphocytes were resuspended in $1 \mathrm{ml}$ of $1 \mathrm{X}$ BD IMag buffer. Ten microliters of the suspension were then mixed with $190 \mu \mathrm{l}$ PBS. Then, $5 \mu \mathrm{l}$ biotinylated human NK cell concentrate was added and incubated in the dark at room temperature for $15 \mathrm{~min}$. Then, $1.8 \mathrm{ml} 1 \mathrm{X}$ BD buffer was added to remove biotin, followed by centrifugation at $300 \mathrm{x} \mathrm{g}$ for $7 \mathrm{~min}$. Finally, the cells were resuspended in $500 \mu \mathrm{l}$ of $1 \mathrm{X}$ BD buffer, followed by addition of equal volume of beads solution. After incubation in the dark for $30 \mathrm{~min}$, the mixture was mixed gently and placed on a magnet for $7 \mathrm{~min}$. The supernatant was then transferred to a new Eppendorf tube, and NK cells were obtained. NK cells were cultured in RPMI-1640 medium supplemented with 10\%
FBS and $100 \mathrm{IU} \mathrm{IL}-2$ at $37^{\circ} \mathrm{C}$ and $5 \% \mathrm{CO}_{2}$ for $48 \mathrm{~h}$ before use.

\section{Quantitative real-time polymerase chain reaction (qRT- PCR)}

Tissue samples $(100 \mathrm{mg})$ were flash frozen in liquid nitrogen, ground, and then lysed with $1 \mathrm{ml}$ TRIzol following the manufacturer's manual (Thermo Fisher Scientific, Waltham, MA, USA). Cells $\left(1 \times 10^{6}\right)$ were directly lysed with $1 \mathrm{ml}$ TRIzol. Total RNA was extracted using phenol chloroform. The concentration and quality of RNA was measured using ultraviolet spectrophotometry (Nanodrop ND2000, Thermo Scientific, Waltham, MA, USA). cDNA was then obtained by reverse transcription of $1 \mu \mathrm{g}$ RNA and stored at $-20^{\circ} \mathrm{C}$. Reverse transcription of mRNA was performed using TIANScript II cDNA First Strand Synthesis Kit (Tiangen, Beijing, China), and reverse transcription of miRNA was carried out using miRcute miRNA cDNA First Strand Synthesis Kit (Tiangen, Beijing, China) . SuperReal PreMix (SYBR Green) qRT-PCR kit (Tiangen, Beijing, USA) was used to detect mRNA expression of HIG2, using GAPDH as an internal reference. The primer sequences of HIG2 were $5^{\prime}$ - ACGAGGGCGCTTTTGT CTC - 3' (forward) and 5' - AGCACAGCATACACCAGACC $-3^{\prime}$ (reverse). The primer sequences of GAPDH were $5^{\prime}$ - CGGAGTCAACGGATTTGGTCGTAT - 3' (forward) and 5' - AGCCTTCTCCATGGTGGTGAAG AC $-3^{\prime}$ (reverse). The reaction $(20 \mu \mathrm{l})$ was composed of $10 \mu \mathrm{l} \mathrm{SYBR}$ Premix EXTaq, $0.5 \mu \mathrm{l}$ upstream primer, $0.5 \mu \mathrm{l}$ downstream primer, $2 \mu \mathrm{l} \mathrm{cDNA}$ and $7 \mu \mathrm{lddH_{2 }}$ O. PCR conditions were: initial denaturation at $95^{\circ} \mathrm{C}$ for $10 \mathrm{~min}$; denaturation at $95^{\circ} \mathrm{C}$ for $1 \mathrm{~min}$ and annealing at $60^{\circ} \mathrm{C}$ for 30 s (40 cycles; iQ5; Bio-Rad, Hercules, CA, USA). The $2^{-\triangle \Delta C \mathrm{q}}$ method [18] was used to calculate the expression of HIG2 mRNA relative to GAPDH. Each sample was tested in triplicate.

\section{CCK-8 assay}

HepG2 and SMMC-7721 cells were seeded at a density of 2000/well in 96-well plates. At 0, 24, 48, and $72 \mathrm{~h}$, $20 \mu \mathrm{l}$ CCK -8 reagent ( $5 \mathrm{~g} / \mathrm{L}$; Beyotime, Shanghai, China) was added to the cells. At the designated time points, $150 \mu \mathrm{l}$ CCK- 8 reaction solution was added, and the cells were incubated at $37^{\circ} \mathrm{C}$ for $2 \mathrm{~h}$. Then, the absorbance of the cells in each well was measured at $490 \mathrm{~nm}$ for 
plotting cell proliferation curves. Each group was tested in three replicate wells, and the values were averaged.

\section{Transwell assay}

Growth factor-depleted Matrigel invasion chambers (BD Biosciences, Franklin Lakes, NJ, USA) were used to measure cell invasion. Matrigel was thawed at $4{ }^{\circ} \mathrm{C}$ overnight and diluted with serum-free DMEM (dilution 1:2). The mixture $(50 \mu \mathrm{l})$ was evenly distributed into the upper chamber (Merck Millipore, Billerica, MA, USA) and incubated at $37^{\circ} \mathrm{C}$ for $1 \mathrm{~h}$. After solidification, $1 \times$ $10^{5}$ cells from each group were seeded into the upper chamber containing $200 \mu \mathrm{l}$ of serum-free DMEM. In addition, $500 \mu \mathrm{l}$ DMEM supplemented with $10 \%$ fetal bovine serum was added to the lower chamber. After 24 $\mathrm{h}$, the chamber was removed, and the cells in the upper chamber were wiped off. After being fixed with $4 \%$ formaldehyde for $10 \mathrm{~min}$, the membrane was stained using Giemsa method for microscopic observation of five random fields $(200 \times)$. For migration array, the tumor cells were seeded onto the upper chamber (Merck Millipore, Billerica, MA, USA) without matrigel, and the rest of the steps were the same as the invasive array. The number of motile cells was calculated to evaluate cell invasion and migration. All procedures were carried out on ice with pipetting tips being cooled to $4{ }^{\circ} \mathrm{C}$.

\section{Western blotting}

Before lysis, tissues $(100 \mathrm{mg})$ were ground into powder, and cells $\left(1 \times 10^{6}\right)$ were trypsinized and collected. Tissue samples or cells were then lysed with chilled radioimmunoprecipitation assay (RIPA) lysis buffer $(600 \mu \mathrm{l}$; $50 \mathrm{mM}$ Tris-base, $1 \mathrm{mM}$ EDTA, $150 \mathrm{mM} \mathrm{NaCl}, 0.1 \%$ sodium dodecyl sulfate, $1 \%$ TritonX-100, $1 \%$ sodium deoxycholate; Beyotime Institute of Biotechnology, Shanghai, China) for $30 \mathrm{~min}$ on ice. The mixture was centrifuged at $12,000 \mathrm{rpm}$ and at $4{ }^{\circ} \mathrm{C}$ for $10 \mathrm{~min}$. The supernatant was used to determine protein concentration by bicinchoninic acid (BCA) protein concentration determination kit (RTP7102, Real-Times Biotechnology Co., Ltd., Beijing, China). The samples were then mixed with $5 \times$ sodium dodecyl sulfate loading buffer before denaturation in boiling water bath for $10 \mathrm{~min}$. Afterwards, the samples $(20 \mu \mathrm{g})$ were subjected to $10 \%$ sodium dodecyl sulfate-polyacrylamide gel electrophoresis at $100 \mathrm{~V}$. The resolved proteins were transferred to polyvinylidene difluoride membranes on ice $(250 \mathrm{~mA}, 1 \mathrm{~h})$ and blocked with $5 \%$ skimmed milk at room temperature for $1 \mathrm{~h}$. The membranes were then incubated with mouse antihuman HIG2 (1:1000; ab78349; Abcam, Cambridge, UK), rabbit anti-human CREB (1:800; ab32515; Abcam), rabbit anti-human NF-kB p65 (1:1000; ab16502; Abcam), mouse anti-human STAT3 (1:1000; ab119352; Abcam), rabbit anti-human STAT1 (1:1000; ab30645; Abcam), rabbit anti-human STAT4 (1:800; ab235946; Abcam), rabbit anti-human STAT5 (1:800; ab16276; Abcam), rabbit anti-human STAT6 (1:800; ab44718; Abcam), mouse anti-human p53 (1:800; ab90363; Abcam) or GAPDH (1:4000; ab70699; Abcam) monoclonal primary antibodies at $4{ }^{\circ} \mathrm{C}$ overnight. After extensive washing with phosphate-buffered saline with Tween 205 times for $5 \mathrm{~min}$ each time, the membranes were incubated with goat anti-mouse horseradish peroxidase-conjugated secondary antibody (1:4000; ab6789; Abcam, Cambridge, UK) for $1 \mathrm{~h}$ at room temperature before washing with phosphate-buffered saline with Tween 205 times for 5 min each time. Membranes were then developed with enhanced chemiluminescence detection kit (Sigma-Aldrich, St. Louis, MO, USA) for imaging. Image lab v3.0 software (Bio-Rad, Hercules, CA, USA) was used to acquire and analyze imaging signals. Each target protein was quantified relative to GAPDH protein levels.

\section{Flow cytometry}

According to the manufacturer's manual, $1 \times 10^{5} \mathrm{NK}$ cells were suspended in $100 \mu \mathrm{l}$ DMEM medium before addition of fluorescence-labelled, activated receptors (p30, CD16, p46, and NKG2D), and inhibitory receptors (158b and NKG2A). The cells were then incubated at room temperature in the dark for $15 \mathrm{~min}$ before examination by flow cytometry. For the detection of effector molecules, NK cells were pre-stained with CD3 and CD56 antibodies, followed by addition of fluorescence-labelled GZMB, Perforin, TNF- $\alpha$, and INF- $\gamma$ antibodies. After incubation in the dark at room temperature for $15 \mathrm{~min}$, the cells were washed 3 times with cold PBS before centrifugation at $600 \times$ g for 5 min to collect the cells. After resuspending the cells in $200 \mu \mathrm{l}$ cold PBS, flow cytometry was performed.

\section{Determination of tumor-killing effect of NK cells by flow cytometry}

NK cells $\left(2 \times 10^{4}\right)$ were mixed with SMMC-7721 or HepG2 cells at a ratio of $1: 4$, and cultured at $37^{\circ} \mathrm{C}$ and $5 \% \mathrm{CO}_{2}$ overnight. The cell density was then adjusted to $1 \times 10^{5} / 100 \mu \mathrm{l}$ and subjected to flow cytometry analysis using the ANXN V FITC APOPTOSIS DTEC KIT I (BD Biosciences, Franklin Lakes, NJ, USA) following the manufacturer's manual for the detection of apoptosis. Cells with ANNEXIN V-positive values were early apoptotic cells, those with PI-positive values were necrotic cells, and those with double positive values were late apoptotic cells.

\section{Lactate dehydrogenase (LDH) assay}

Cells were seeded in 24-well plates at a density of $2 \times$ $10^{5}$ cells/well, and incubated with serum from healthy subjects, sepsis patients or septic shock patients for $24 \mathrm{~h}$. 


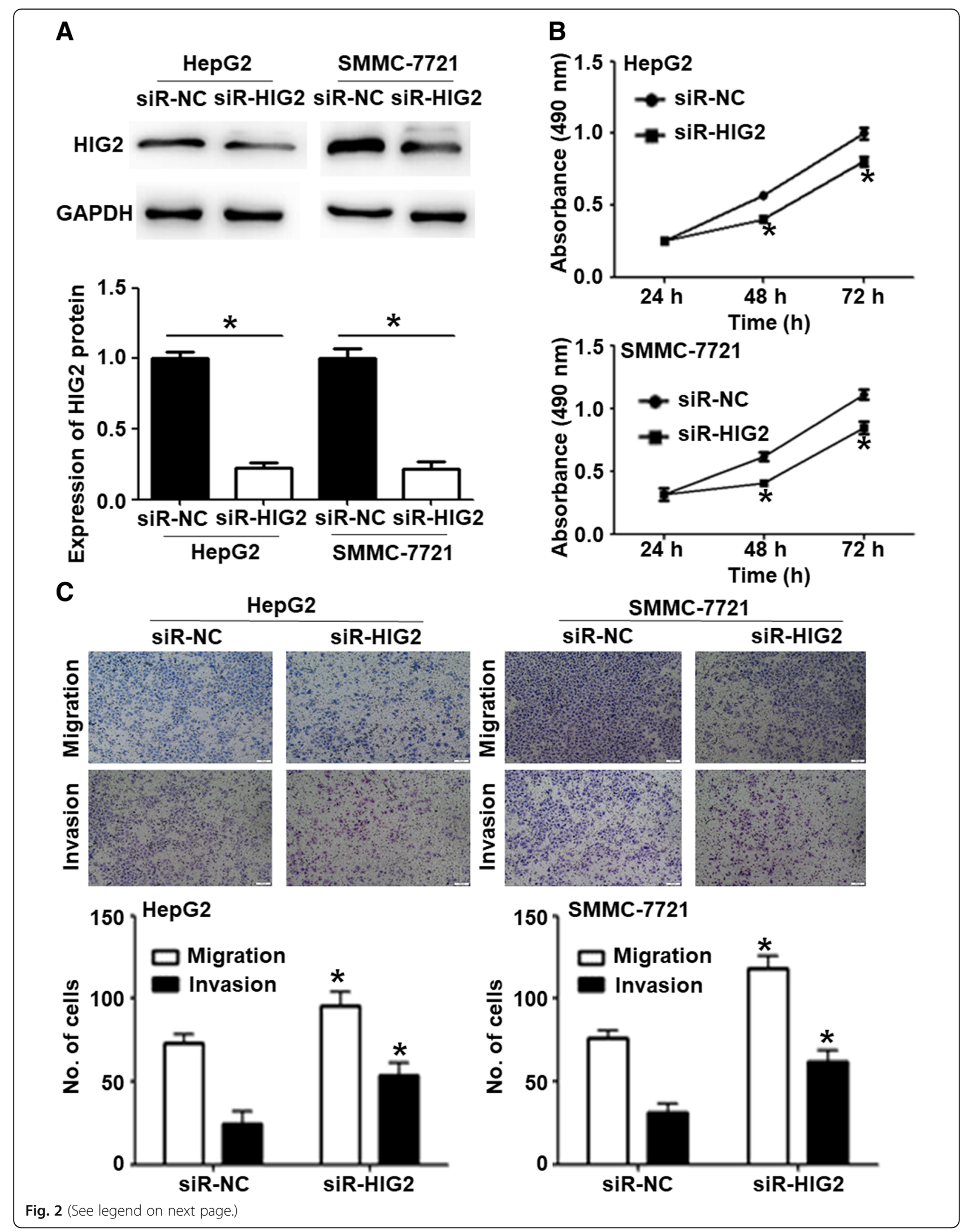


(See figure on previous page.)

Fig. 2 Effect of silencing HIG2 on HCC cell migration and invasion in vitro. a HIG2 protein expression in HepG2 and SMMC-7721 cells transfected with siR-HIG2 or siR-NC, as determined by Western blotting. ${ }^{*} P<0.05$. b Proliferation of HepG2 and SMMC-7721 cells transfected with siR-HIG2 or siR-NC, as determined by CCK-8 assay. ${ }^{*} P<0.05$ compared with siR-NC at the same time points. c Migration and invasion of HepG2 and SMMC7721 cells transfected with siR-HIG2 or siR-NC, as determined by Transwell assay. ${ }^{*} P<0.05$ compared with the respective siR-NC group

Then, the medium was replaced with fresh medium, followed by incubation for $12 \mathrm{~h}$. The supernatant was collected and centrifuged at $12,000 \times \mathrm{g}$ for $10 \mathrm{~min}$. Afterwards, $120 \mu \mathrm{l}$ of supernatant was used for LDH assay following the manufacturer's manual (Beyotime, Shanghai, China).

\section{Tumorigenesis assay in nude mice}

For performing the tumorigenesis assay in vivo, female BALB/c-nu mice (5-6 weeks of age, 16-20 g) were purchased and kept in barrier facilities on a $12 \mathrm{~h}$ light/dark cycle. All experimental procedures were approved by the
Institutional Animal Care and Use Committee of Guangxi Medical University. Briefly, BALB/c-nu mice were injected with $1 \times 10^{6}$ of the indicated cells under the armpit (tumor cells were suspended in $200 \mu \mathrm{l}$ sterile PBS). Six weeks later, all mice were euthanized, and tumors were dissected and sectioned ( $4 \mu \mathrm{m}$ in thickness), followed by H\&E staining or IHC.

\section{Metastasis assay in nude mice}

For pulmonary metastasis assays, the nude mice were divided into 2 groups, siR-NC and siR-HIG2. Two million

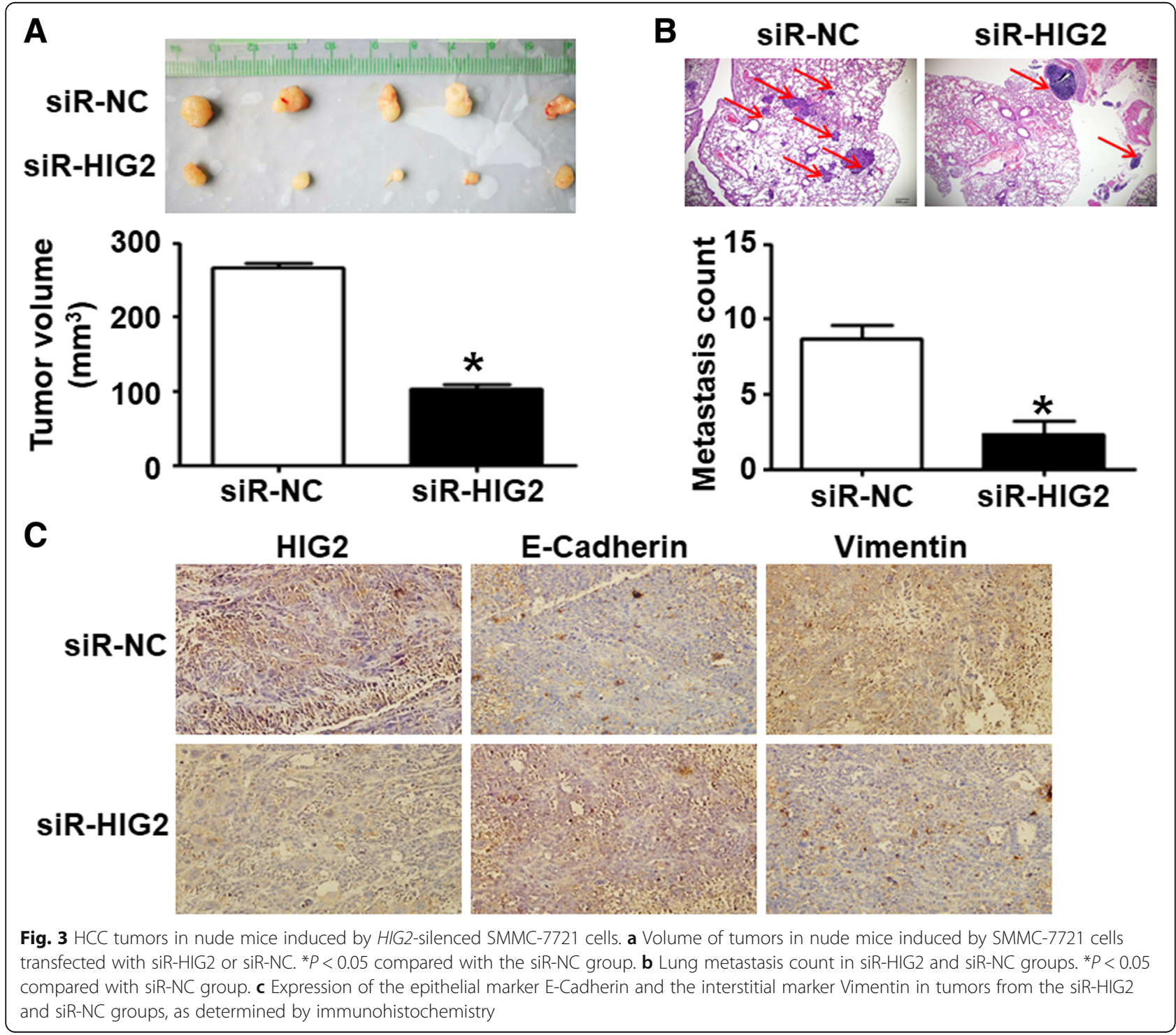


SMMC-7721 cells transfected with siR-HIG2 or siR-NC were suspended in $200 \mu \mathrm{l}$ phosphate-buffered saline for each mouse. The indicated tumor cells were injected into nude mice (6 per group, 5-week-old) through the lateral tail vein. After 6 weeks, the mice were euthanized and each lung was dissected and fixed with phosphatebuffered neutral formalin before paraffin embedment.
The paraffin blocks were then cut into five sections and stained with H\&E. We then observed the sections under a light microscope for calculating the metastatic nodules.

\section{Dual-luciferase reporter assay}

To investigate whether CREB protein could directly bind to the promoter region of IL-10, dual-luciferase reporter

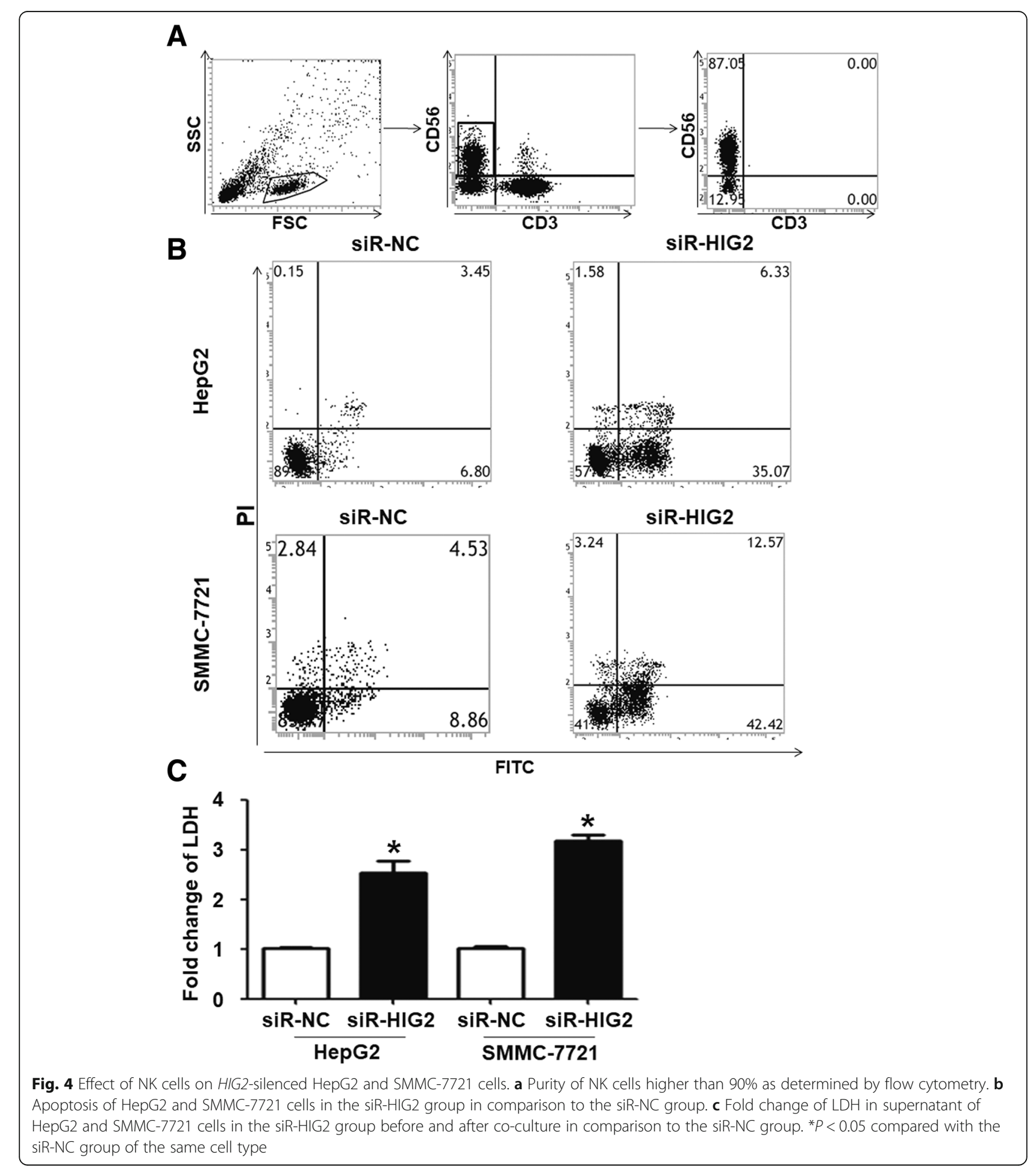


assay was performed in vitro. In brief, the promoter sequence of the $I L-10$ gene was predicted in silico (http:// biogrid-lasagna.engr.uconn.edu) and amplified by qRTPCR. The primers were as follows: 5 '-AGGAGAAGTCT TGGGTATTCATCC-3' (forward) and 5'-AAGCCCCT GATGTGTAGACC-3' (reverse). The plasmid harboring the TSS sequence of CREB (pcDNA3.1-CREB) was constructed by Genechem Co. Ltd. (Shanghai, China). The promoter sequence of $I L-10$ was cloned into the luciferase reporter plasmid pGL6 (Beyotime, Beijing, China) that contained XhoI or HindIII restriction sites. $293 \mathrm{~T}$ cells were transfected with the reporter plasmid together with pcDNA3.1-CREB using liposome method. After 24h of incubation, the cells from each group were lysed according to the manufacturer's instructions (Beyotime, Beijing, China). Luminescence intensity was recorded by a GloMax 20/20 luminometer (Promega Corp., Fitchburg, WI, USA). Luminescence activity of Renilla luciferase was used as the internal reference, and cell luminescence values in each group were statistically analyzed.

\section{Statistical analysis}

Continuous variables are represented by mean \pm standard deviation. The comparison between two groups was performed by using student's $t$ test. A $p$-value less than 0.05 was considered statistically significant. ANOVA followed by a post hoc multiple comparisons test was used for the comparison of multiple groups. All experiments were repeated three times.

\section{Results}

\section{HIG2 expression is upregulated in HCC}

The GEPIA database was used to perform a preliminary assessment of the association between the expression of HIG2 gene in HCC tissues and prognosis of HCC. The search results showed that the level of the HIG2 gene in HCC tissues was significantly higher than that in tumoradjacent tissues (Fig. 1a). Postoperative survival analysis showed that the 5-year survival and disease-free survival rates of $\mathrm{HCC}$ patients with high expression of HIG2 were lower than those of $\mathrm{HCC}$ patients with low expression of HIG2 (Fig. 1b and c). Our data showed that HIG2 mRNA expression in HCC tissues was significantly higher than that in tumor-adjacent tissues $(P<0.05)$ (Fig. 1d). In addition, HIG2 expression in tumor tissues from HCC patients with lymph node metastasis was significantly higher than that from HCC patients without lymph node metastasis $(P<0.05)$ (Fig. 1e). HIG2 expression in tumor tissues from HCC patients with TNM Stage III/IV disease was significantly higher than that in tumor tissues from HCC patients with TNM Stage I/II disease $\quad(P<0.05) \quad$ (Fig. 1f). Immunohistochemistry showed that HIG2 expression was detected in the majority of the tested HCC tissues (35/40), and only in a small number of tumor-adjacent tissues (2/40) (Fig. $1 \mathrm{~g}$ and $\mathrm{h}$ ). Consistently, the expression of HIG2 mRNA in HepG2 and SMMC-7721 cells was significantly higher than that in tumor-adjacent tissues $(P<0.05$; Fig. 1i). These results suggest that HIG2 expression is upregulated in HCC.

\section{Silencing of HIG2 suppresses HCC cell migration and invasion in vitro and in vivo}

To further study the function of the HIG2 gene in HCC, we used siR-HIG2 to downregulate the expression of HIG2 in HepG2 and SMMC-7721 cells. Western blotting showed that HIG2 protein levels in HepG2 and SMMC-7721 cells transfected with siR-HIG2 were significantly lower than that in the cells transfected with siR-NC $(P<0.05)$ (Fig. 2a). CCK-8 assay showed that the proliferation of HepG2 and SMMC-7721 cells transfected with siR-HIG2 was significantly reduced in comparison to the proliferation of cells transfected with siRNC $(P<0.05)$ (Fig. 2b). In addition, Transwell assay showed that the number of migratory HepG2 and SMMC-7721 cells in the siR-HIG2 group was significantly lower than those in the siR-NC group $(P<0.05)$ (Fig. 2c). We then generated nude mouse models of tumor formation and lung metastasis using SMMC-7721 cells. The results showed that the mean volume of tumors induced by SMMC-7721 cells transfected with siRHIG2 were significantly smaller than that of tumors induced by SMMC-7721 cells transfected with siR-NC ( $P$ $<0.05$ ) (Fig. 3a). Additionally, a smaller number of metastatic foci were observed in the siR-HIG2 group in comparison to the siR-NC group $(P<0.05)$ (Fig. 3b). Immunohistochemical analysis showed that the epithelial marker E-Cadherin was upregulated in tumors from the siR-HIG2 group, while the expression of the interstitial marker Vimentin was downregulated in tumors from the siR-HIG2 group. This suggests that the epithelial-tomesenchymal transition (EMT) in the siR-HIG2 group was enhanced (Fig. 3c). The results indicate that silencing of HIG2 suppresses HCC cell migration and invasion in vitro and in vivo.

\section{The killing effect of NK cells on HepG2 and SMMC-7721 cells is enhanced after HIG2 silencing in HepG2 and SMMC-7721 cells}

To determine the effect of HIG2 expression on the killing of HCC cells by NK cells, HepG2 and SMMC-7721 cells were transfected with siR-NC and siR-HIG2 and co-cultured with NK cells. Flow cytometry analysis showed that the purity of NK cells was more than $90 \%$, surpassing the purity threshold required for our experiments (Fig. 4a). After being co-cultured with NK cells, apoptosis of HepG2 and SMMC-7721 cells in the siRHIG2 group was enhanced in comparison to that in the siR-NC group (Fig. 4b). The fold change of lactate 


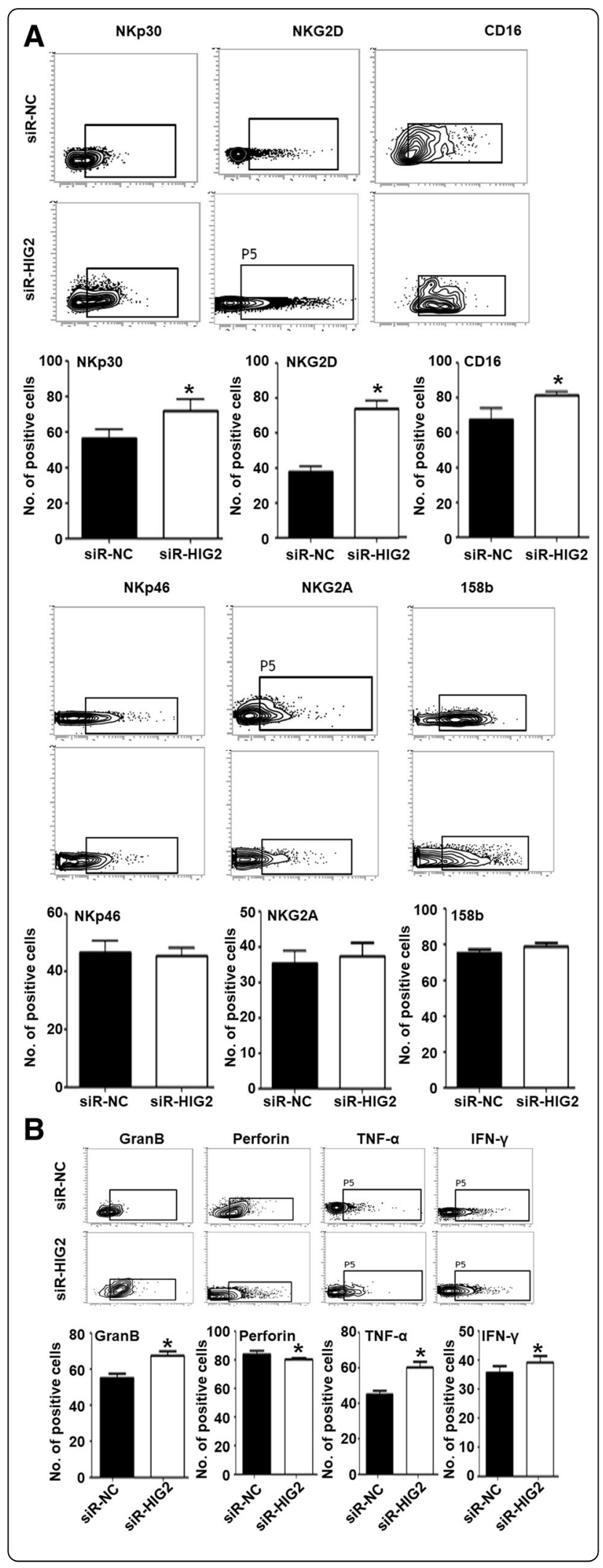

Fig. 5 Effect of conditioned media from HIG2-silenced SMMC-7721 cells on the phenotype and function of NK cells. a Ratio of NK cells with positive expression of NKp30, NKG2D, CD16, NKp46, NKG2A, or 158b after treatment with the conditioned media of SMMC-7721 cells in the siR-HIG2 or siR-NC group. ${ }^{*} P<0.05$ compared with the siR-NC group. $\mathbf{b}$ Ratio of NK cells with positive expression of Granzyme B, Perforin, TNF-a, or IFN- $\gamma$ after treatment with the conditioned media of SMMC-7721 cells in the SiR-HIG2 or siR-NC group. ${ }^{*} P<0.05$ compared with the siR-NC group

dehydrogenase (LDH) in the conditioned media of HepG2 and SMMC-7721 cells in the siR-HIG2 group before and after co-culture was significantly higher than that in the siR-NC group $(P<0.05)$ (Fig. 4c). The results suggest that the killing effect of NK cells on HepG2 and SMMC-7721 cells is enhanced after silencing HIG2 expression in HepG2 and SMMC-7721 cells.

\section{Conditioned media of HIG2-silenced SMMC-7721 cells inhibits the phenotype and function of NK cells}

To study the mechanism by which HIG2 promoted the escape of HCC from killing by NK cells, we treated NK cells with the conditioned media of SMMC-7721 cells in the siR-NC and siR-HIG2 groups. Flow cytometry analysis showed that after treatment with the conditioned media of SMMC-7721 cells in the siR-HIG2 group, the proportion of NK cells with positive expression of NKG2D, NKp30, and CD16 was significantly upregulated $(P<0.05)$, while the proportion of NK cells with positive expression of NKp46, NKG2A, or $158 \mathrm{~b}$ was not altered $(P>0.05)$ (Fig. 5a). After treatment with the conditioned media of SMMC-7721 cells in the siR-HIG2 group, the proportion of NK cells with positive expression of Granzyme B (GZMB) and TNF- $\alpha$ was significantly higher $(P<0.05)$, while the proportion of NK cells with positive expression of perforin or IFN- $\gamma$ was not altered $(P>0.05)$ (Fig. 5b). These results indicate that of the conditioned media of HIG2-silenced HCC cells inhibits the phenotype and function of NK cells.

HIG2-silenced HepG2 and SMMC-7721 cells modulate the activity of NK cells through the STAT3 signaling pathway To understand how HIG2 modulates the activity of NK cells, changes in the STAT signaling pathway were examined by flow cytometry. The data showed that phosphorylation levels of STAT1 and STAT4 in NK cells treated with the conditioned media of SMMC-7721 cells in the siR-HIG2 group were significantly higher than those in the siR-NC group $(P<0.05)$. The phosphorylation level of STAT3 in NK cells treated with the conditioned media of SMMC-7721 cells in the siR-HIG2 group was significantly lower than that in the siR-NC group $(P<$ 0.05). Additionally, the phosphorylation level of STAT5 


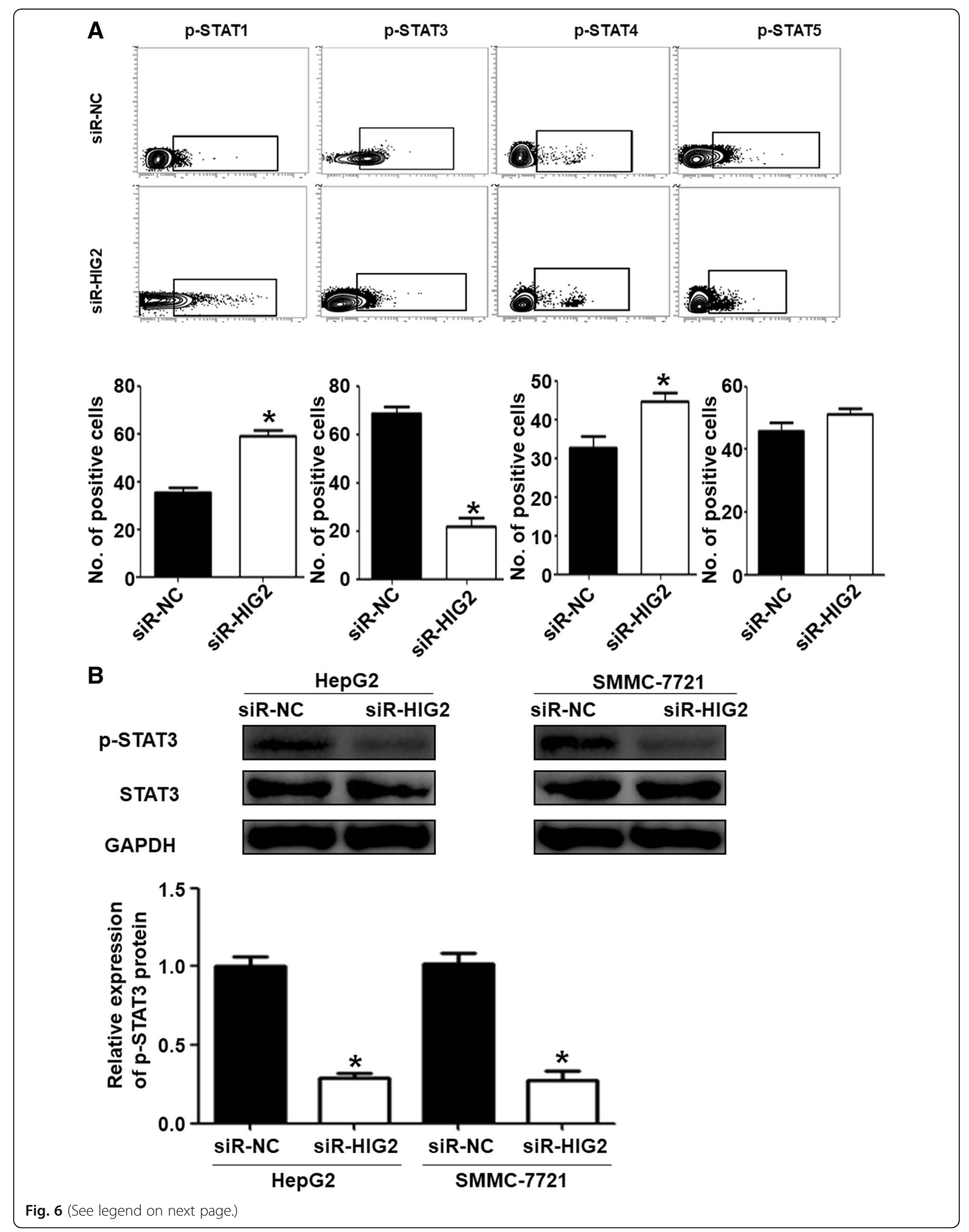


(See figure on previous page.)

Fig. 6 HIG2-silenced HepG2 and SMMC-7721 cells modulate the activity of NK cells through the STAT3 signaling pathway. a Phosphorylation levels of STAT1, STAT3, STAT4, and STAT5 in NK cells treated with the conditioned media of SMMC-7721 cells in the siR-HIG2 or siR-NC group, as determined by flow cytometry. ${ }^{*} P<0.05$ compared with the siR-NC group. $\mathbf{b}$ Expression of phosphorylated STAT3 protein in NK cells treated with the conditioned media of HepG2 or SMMC-7721 cells in the siR-HIG2 or siR-NC group, as determined by Western blotting. ${ }^{*} P<0.05$ compared with siR-NC of the same cell type

in NK cells treated with the conditioned media of SMMC-7721 cells in the siR-HIG2 group was not significantly different from that in the siR-NC group $(P>$ 0.05) (Fig. 6a). Western blotting showed that the expression of phosphorylated STAT3 in NK cells treated with the conditioned media of HepG2 or SMMC-7721 cells in the siR-HIG2 group was significantly lower than that in the siR-NC group $(P<0.05)$ (Fig. 6b). The results suggest that HIG2-silenced HepG2 and SMMC-7721 cells can modulate the activity of NK cells through the STAT3 signaling pathway.

\section{HIG2 gene promotes the evasion of HCC cells from killing} by NK cells through upregulation of IL-10

Increasing evidence revealed that IL10 is one of the key negative regulators of NK cell activity through STAT3 pathway. To test whether HIG2 can help HCC cells escape immune surveillance of NK cells through IL-10, we co-cultured NK cells with IL-10-containing conditioned media of HepG2 and SMMC-7721 cells. Immunohistochemistry analysis showed that IL-10 protein expression in HCC tissues was higher than that in tumor-adjacent tissues (Fig. 7a). qRT-PCR and ELISA data showed that IL-10 mRNA expression in HepG2 and SMMC-7721 cells transfected with siR-HIG2 was significantly lower than that in the siR-NC group $(P<0.05)$ (Fig. 7b and $\mathrm{c}$ ). Flow cytometry analysis showed that the apoptotic rates of HepG2 and SMMC-7721 cells in the siR-HIG2 + IL10 group were significantly higher than those in the siRNC group $(P<0.05)$ but were significantly lower than those in the siR-HIG2 group $(P<0.05)$ (Fig. 7d). After treatment with IL-10, the proportion of NK cells with positive expression of NKp30 and NKG2D receptors was significantly higher than that of the NC group $(P<0.05)$ (Fig. 7e), but the proportion of NK cells with positive expression of CD16, GZMB, or TNF- $\alpha$ was not significantly different from that of the NC group $(P>0.05)$ (Fig. 7f). The results indicate that HIG2 promotes the evasion of HCC cells from killing by NK cells through upregulation of IL-10.

\section{HIG2 promotes IL-10 expression through the AMPK/CREB signaling pathway}

To further investigate the underlying mechanism by which HIG2 regulates IL-10 expression in HCC cells, we performed bioinformatic analysis to identify the signaling pathway that regulates IL-10 expression. Our results revealed that several transcription factors (TFs), which are involved in the AMPK, NF-kB, and STAT signaling pathways, may directly bind to the promoter of the $I L-10$ gene (Fig. 8a). Furthermore, we determined the expression of the TFs in the nuclei of the indicated HCC cells by Western blotting and found that CREB expression was significantly inhibited in the nuclei of HIG2-silenced HCC cells. However, the expression of other TFs showed no significant changes (Fig. 8b-d). Next, we overexpressed CREB protein in HIG2-silenced HCC cells (Fig. 8e) and found that CREB not only restored the phenotype of HIG2-silenced HCC cells but also increased the expression of IL10 (Fig. 8f-h). These observations indicate that HIG2 regulated IL-10 expression via CREB. CREB is a well-known downstream factor of AMPK signaling. Therefore, we investigated the activation of the AMPK pathway. The results revealed that phospho-AMPK $\alpha$ (Thr172) was suppressed in HIG2-silenced HCC cells (Fig. 9a and b). Additionally, we confirmed that metformin hydrochloride, an activator of AMPK signaling, could restore IL-10 expression in HIG2-silenced HCC cells (Fig. 9c). Dual luciferase reporter assay also revealed that CREB protein could enhance the transcriptional activity of IL-10 (Fig. 9d). These results indicate that the HIG2 gene promotes IL-10 expression through the AMPK/CREB signaling pathway.

\section{Discussion}

At present, recurrence and metastasis are key factors that limit clinical outcomes of $\mathrm{HCC}$ patients [3]. Immune escape is also an important prerequisite for tumor recurrence and metastasis [19]. NK cells are important cells in the innate immune system, and they can quickly identify and kill tumor cells [18, 20]. The activation of multiple oncogenes increases the metastatic ability of tumor cells, but the effect of these genes on the immunological properties of tumor cells is unclear.

As a specific downstream target gene of HIF-1, HIG2 plays an important role in the activation of the hypoxiainduced signaling pathway, which is closely related to the proliferation and metastasis of tumor cells [21]. Using bioinformatics, we discovered that the overall and disease-free survival rates of HCC patients with high expression of HIG2 were significantly lower than those of HCC patients with low expression of HIG2, indicating that the expression level of HIG2 is of clinical 


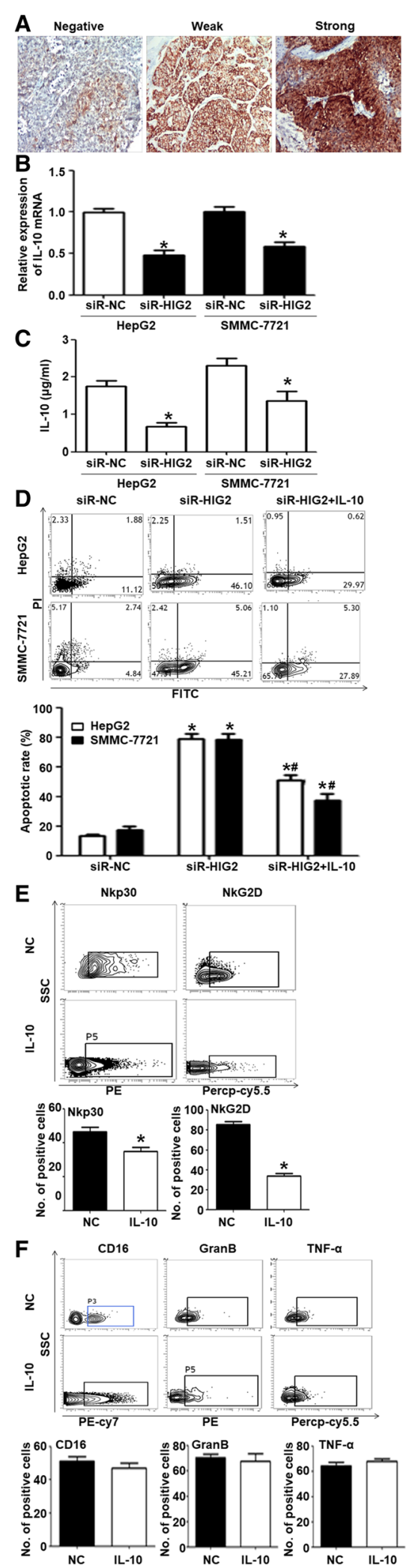

Fig. 7 HIG2 gene promotes the evasion of HCC cells from killing by NK cells through upregulation of IL-10 expression. a IL-10 protein expression in HCC tissues or tumor-adjacent tissues, as determined by immunohistochemistry. $\mathbf{b}$ and $\mathbf{c} / L-10$ mRNA expression or secreted IL-10 protein in HepG2 and SMMC-7721 cells transfected with siR-HIG2 or siR-NC, as determined by qRT-PCR and ELISA, respectively. ${ }^{*} P<0.05$ compared with the siR-NC group of the same cell type. $\mathbf{d}$ Apoptotic rates of HepG2 and SMMC-7721 cells in the siR-NC, siR-HIG2, and siR-HIG2 + IL-10 groups. ${ }^{*} P<0.05$ compared with the siR-NC group; \#P<0.05 compared with the siR-HIG2 + IL-10 group. e Proportion of NK cells with positive expression of NKp30 and NKG2D receptors after treatment with $\mathrm{IL}-10 .{ }^{*} P<0.05$ compared with the NC group. $\mathbf{f}$ Proportion of NK cells with positive expression of CD16, GZMB, or TNF-a after treatment with IL-10

significance for the prognosis of HCC patients. qRT-PCR results showed that the expression of HIG2 was significantly upregulated in HCC tissues, and positively correlated with lymph node metastasis and TNM stage, suggesting that HIG2 is associated with the occurrence and development of HCC. Immunohistochemical analysis showed that the positive expression of HIG2 protein in HCC tissues was significantly higher than that in tumoradjacent tissues. Elevated levels of HIG2 were also observed in HCC cell lines HepG2 and SMMC-7221. After interfering with the expression of HIG2 in HepG2 and SMMC-7721 cells, the proliferation, migration, and invasion were significantly inhibited, indicating that HIG2 may function as an oncogene in HCC.

Increasing evidence shows that numerous lymphocytes infiltrate tumor tissues to inhibit tumor growth and metastasis [22, 23]. Therefore, evading killing by immune cells is one of the key factors driving tumor cell survival. Tumor cells can promote immune evasion by altering their immunogenicity and regulating the activity of immune cells [24]. For example, tumor cells can escape killing by NK cells through autocrine downregulation of the expression of $\mathrm{MICA} / \mathrm{B}$ protein [25]. In addition, tumor cells can induce macrophage differentiation into tumor associated macrophage type 2 (TAM2), thereby promoting tumor cell immune escape [26]. In the present study, after interfering with HIG2 gene expression, the killing effect of NK cells on HCC cells was significantly enhanced. There was also an appreciable fold increase in LDH release from HIG2-silenced cells in comparison with controls, suggesting that HIG2 helps HCC cells escape NK cell-mediated cytotoxicity. Our flow cytometry results showed that conditioned media of HIG2-silenced HCC cells stimulated the expression of the activated receptor NKp30, NKG2D, and CD16 on NK cells, and upregulated the expression of the effector molecules GZMB, Perforin, TNF- $\alpha$, and IFN- $\gamma$. These data suggest that HIG2- 


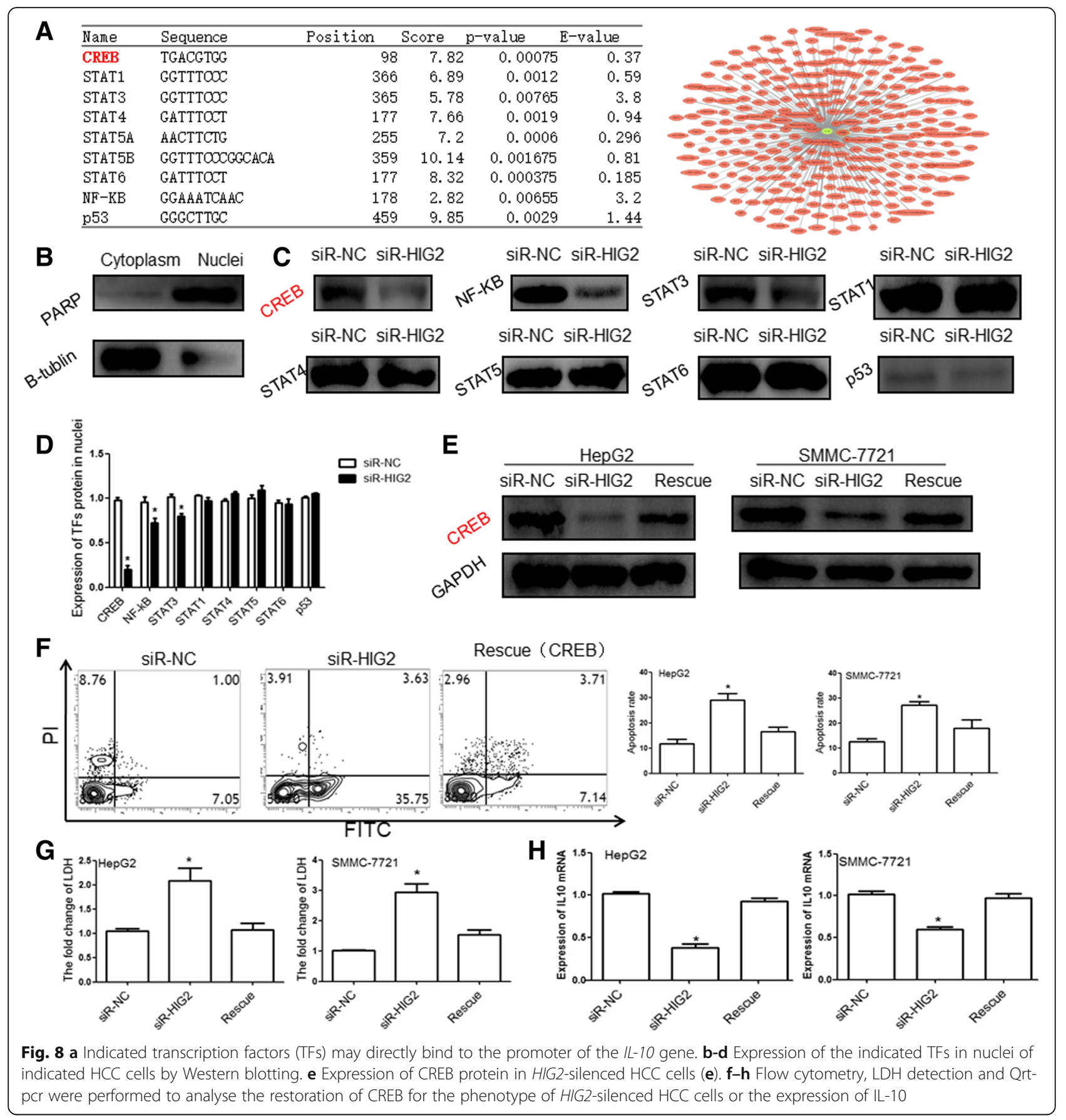

silenced HCC cells can enhance the killing activity of NK cells.

The STAT signaling pathway plays an important regulatory role in NK cell differentiation, maturation, and activation. For example, STAT1 and STAT2 can activate NK cells, while STAT3 inhibits NK cell activity $[27,28]$. Our results showed that the conditioned media of HIG2-silenced HCC cells significantly reduced the phosphorylation level of STAT3, but only slightly elevated the phosphorylation levels of STAT1 and STAT4 proteins, which can promote the activity of NK cells. Therefore, we hypothesize that the conditioned media of HIG2-silenced HCC cells can upregulate the activity of NK cells by inhibiting intracellular STAT3 signaling.

Studies have shown that the STAT3 signaling pathway in NK cells is regulated by many cytokines such 


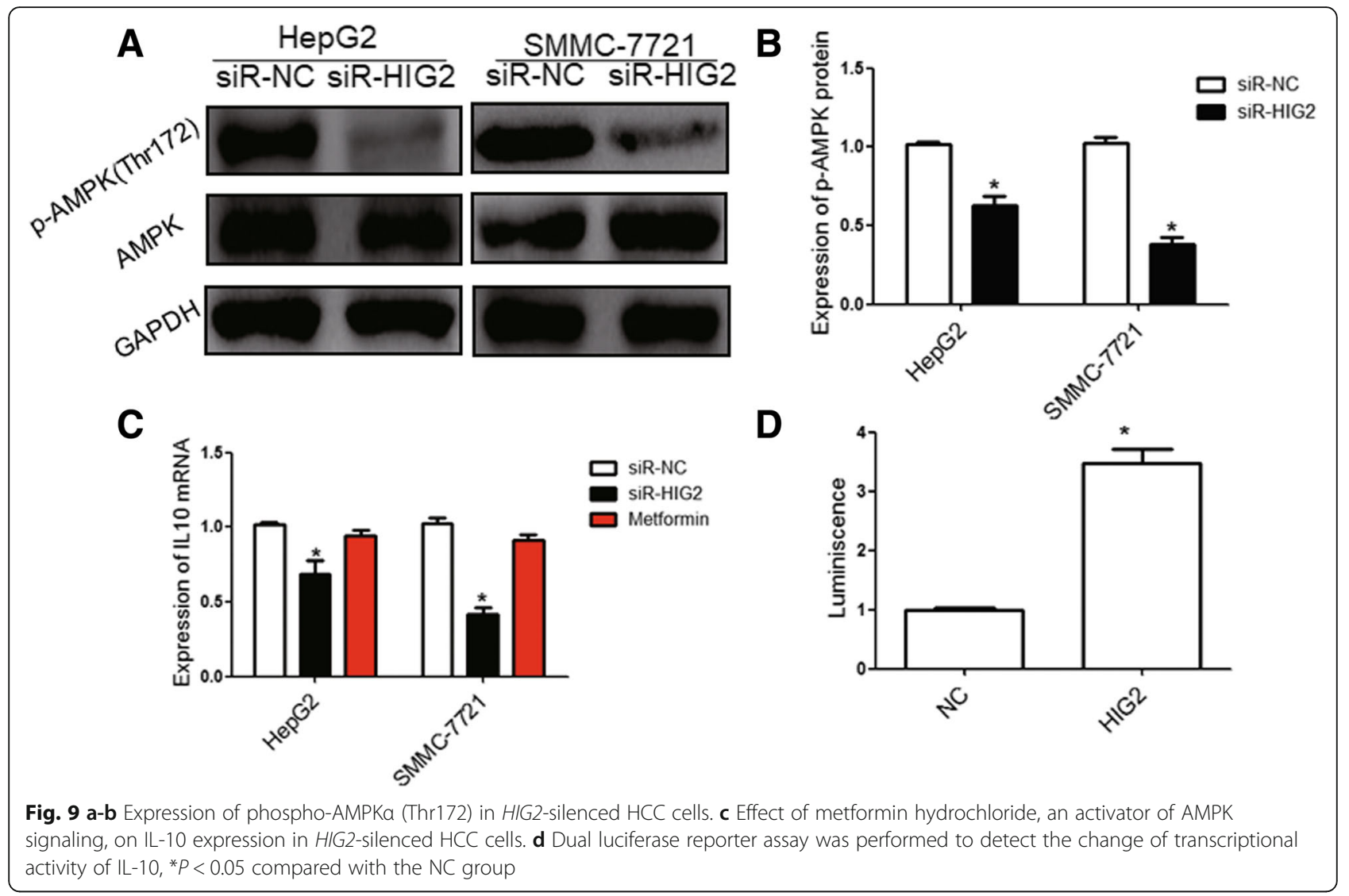

as IL10, IL-12, and IL-15 [29]. Among these, the effect of IL-10 on the activation of the STAT3 pathway is the most significant. In the present study, we found that IL-10 protein expression in HCC tissues was significantly higher than that in tumor-adjacent tissues, and IL-10 mRNA levels in HIG2-silenced HCC cells were significantly decreased. Additionally, treatment with IL-10 protein significantly restored the cytotoxic capacity of NK cells, which had been inhibited by HIG2-silenced HCC cells. Flow cytometry showed that treatment with an IL-10 antibody significantly up-regulated the expression of activated receptors NKp30 and NKG2D on the surface of NK cells and down-regulated the expression of p-STAT3 in NK cells, suggesting that HIG2 induced the activation of the STAT3 signaling pathway in NK cells by up-regulation of IL-10 expression in HCC cells. Consequently, the tumor killing activity of NK cells was reduced, promoting the metastasis of HCC cells. Mechanistically, we found that CREB can enhance the transcriptional activity of IL-10 and confirmed that HIG2 can increase the expression of p-AMPK $\alpha$ and CREB nuclear import. These data indicate that HIG2 can increase IL-10 expression through the AMPK/CREB signaling pathway.

\section{Conclusion}

The present study demonstrates that the HIG2 gene is highly expressed in HCC and is closely related to tumor progression and prognosis. Mechanistically, HIG2 increases IL-10 expression via AMPK/CREB signaling, and the secreted IL-10 inhibits the cytotoxicity of NK cells through the STAT3 signaling pathway, thereby promoting the recurrence and metastasis of HCC.

\section{Abbreviations}

DAB: Diaminobenzidine; FBS: Fetal bovine serum; GEPIA: Gene Expression Profiling Interactive Analysis; HCC: Hepatocellular carcinoma; HIF-1: Hypoxiainducible factor-1; HIG2: Hypoxia-inducible gene 2; LD: Lipid droplet; LDH: Lactate dehydrogenase; NK: Natural killer; PBS: Phosphate-buffered saline; qRT-PCR: Quantitative real-time polymerase chain reaction

\section{Acknowledgments}

The authors wish to thank their department and research team for their help and dedication. We thank LetPub (www.letpub.com) for its linguistic assistance during the preparation of this manuscript.

\section{Funding}

This work was supported in part by the National Natural Science Foundation of China (Grant No. 81460512).

Availability of data and materials

The datasets used and/or analyzed during the current study are available from the corresponding author on reasonable request. 


\section{Authors' contributions}

The final version of the manuscript has been read and approved by all authors, and each author believes that the manuscript represents honest work. CC and KF collaborated to design the study. CC, KF, LY and ZC were responsible for performing experiments. $\mathrm{CC}, \mathrm{KF}, \mathrm{QH}$ and $\mathrm{XM}$ analyzed the data. All authors collaborated to interpret results and develop the manuscript.

\section{Ethics approval and consent to participate}

All procedures performed in the current study were approved by the Ethics Committee of Chongqing Cancer Hospital. Written informed consent was obtained from all patients or their families.

\section{Consent for publication}

Written informed consents for publication of any associated data and accompanying images were obtained from all patients or their parents, guardians or next of kin.

\section{Competing interests}

The authors declare that they have no competing interests.

\section{Publisher's Note}

Springer Nature remains neutral with regard to jurisdictional claims in published maps and institutional affiliations.

\section{Author details}

'Department of Epidemiology, Guangxi Medical University, No. 22 Shuangyong Road, Nanning 530021, Guangxi Zhuang Autonomous Region, People's Republic of China. '2Department of Pathology, Chongqing University Cancer Hospital, Chongqing, People's Republic of China.

\section{Received: 1 March 2019 Accepted: 15 May 2019}

Published online: 29 May 2019

\section{References}

1. Delire $B$, Henriet $P$, Lemoine $P$, Leclercq IA, Starkel P. Chronic liver injury promotes hepatocarcinoma cell seeding and growth, associated with infiltration by macrophages. Cancer Sci. 2018;109(7):2141-52.

2. Hassany M, Elsharkawy A, Maged A, Mehrez M, Asem N, Gomaa A, et al. Hepatitis $C$ virus treatment by direct-acting antivirals in successfully treated hepatocellular carcinoma and possible mutual impact. Eur J Gastroenterol Hepatol. 2018;30(8):876-81

3. Chen L, Guo P, He Y, Chen Z, Chen L, Luo Y, et al. HCC-derived exosomes elicit HCC progression and recurrence by epithelial-mesenchymal transition through MAPK/ERK signalling pathway. Cell Death Dis. 2018:9(5):513.

4. Kuzuya T, Ishigami M, Ishizu Y, Honda T, Hayashi K, Ishikawa T, et al. Prognostic factors associated with Postprogression survival in advanced hepatocellular carcinoma patients treated with Sorafenib not eligible for second-line Regorafenib treatment. Oncology. 2018;95(2):91-9.

5. Goto K, Arai J, Stephanou A, Kato N. Novel therapeutic features of disulfiram against hepatocellular carcinoma cells with inhibitory effects on a disintegrin and metalloproteinase 10. Oncotarget. 2018;9(27):18821-31.

6. Zhang $M$, Pang HJ, Zhao W, Li YF, Yan LX, Dong ZY, et al. VISTA expression associated with CD8 confers a favorable immune microenvironment and better overall survival in hepatocellular carcinoma. BMC Cancer. 2018;18(1):511.

7. Maier A, Wu H, Cordasic N, Oefner P, Dietel B, Thiele C, et al. Hypoxiainducible protein $2 \mathrm{Hig} 2 /$ Hilpda mediates neutral lipid accumulation in macrophages and contributes to atherosclerosis in apolipoprotein Edeficient mice. FASEB J. 2017;31(11):4971-84.

8. Knight M, Braverman J, Asfaha K, Gronert K, Stanley S. Lipid droplet formation in mycobacterium tuberculosis infected macrophages requires IFN-gamma/HIF-1alpha signaling and supports host defense. PLoS Pathog. 2018;14(1):e1006874

9. DiStefano MT, Danai LV, Roth Flach RJ, Chawla A, Pedersen DJ, Guilherme A, et al. The lipid droplet protein hypoxia-inducible gene 2 promotes hepatic triglyceride deposition by inhibiting lipolysis. J Biol Chem. 2015;290(24): 15175-84.

10. Dijk W, Mattijssen F, de la Rosa Rodriguez M, Loza Valdes A, Loft A, Mandrup S, et al. Hypoxia-inducible lipid droplet-associated is not a direct physiological regulator of lipolysis in adipose tissue. Endocrinology. 2017; 158(5):1231-51.

11. Obara W, Karashima T, Takeda K, Kato R, Kato Y, Kanehira M, et al. Effective induction of cytotoxic $T$ cells recognizing an epitope peptide derived from hypoxia-inducible protein 2 (HIG2) in patients with metastatic renal cell carcinoma. Cancer Immunol, Immunother : CII. 2017;66(1):17-24.

12. Kuci V, Nordstrom L, Conrotto P, Ek S. SOX11 and HIG-2 are cross-regulated and affect growth in mantle cell lymphoma. Leuk Lymphoma. 2016;57(8): 1883-92.

13. Boraschi $D$, Italiani P. Innate immune memory: time for adopting a correct terminology. Front Immunol. 2018;9:799.

14. Oberg HH, Kellner C, Gonnermann D, Sebens S, Bauerschlag D, Gramatzki M, et al. Tribody [(HER2)2xCD16] is more effective than Trastuzumab in enhancing gammadelta T cell and natural killer cell cytotoxicity against HER2-expressing Cancer cells. Front Immunol. 2018;9:814

15. Roman Aguilera A, Lutzky VP, Mittal D, Li XY, Stannard K, Takeda K, et al. CD96 targeted antibodies need not block CD96-CD155 interactions to promote NK cell anti-metastatic activity. Oncoimmunology. 2018;7(5): e1424677.

16. Zaiatz-Bittencourt V, Finlay DK, Gardiner CM. Canonical TGF-beta signaling pathway represses human NK cell metabolism. J Immunol (Baltimore, Md 1950). 2018;200(12):3934-41.

17. Wang H, Wang L, Cao L, Zhang Q, Song Q, Meng Z, et al. Inhibition of autophagy potentiates the anti-metastasis effect of phenethyl isothiocyanate through JAK2/STAT3 pathway in lung cancer cells. Mol Carcinog. 2018:57(4):522-35.

18. Kaur K, Topchyan P, Kozlowska AK, Ohanian N, Chiang J, Maung PO, et al. Super-charged NK cells inhibit growth and progression of stem-like/poorly differentiated oral tumors in vivo in humanized BLT mice; effect on tumor differentiation and response to chemotherapeutic drugs. Oncoimmunology. 2018;7(5):e1426518.

19. Mo Z, Lu H, Mo S, Fu X, Chang S, Yue J. Ultrasound-guided radiofrequency ablation enhances natural killer-mediated antitumor immunity against liver cancer. Oncol Lett. 2018;15(5):7014-20.

20. Zhang X, Saarinen AM, Hitosugi T, Wang Z, Wang L, Ho TH, et al. Inhibition of intracellular lipolysis promotes human cancer cell adaptation to hypoxia. Elife. 2017:6. https://doi.org/10.7554/eLife.31132.

21. Ji J, Yin Y, Ju H, Xu X, Liu W, Fu Q, et al. Long non-coding RNA Lnc-Tim3 exacerbates CD8 T cell exhaustion via binding to Tim-3 and inducing nuclear translocation of Bat3 in HCC. Cell Death Dis. 2018;9(5):478.

22. Herfs $M$, Roncarati $P$, Koopmansch B, Peulen $O$, Bruyere $D$, Lebeau A, et al. A dualistic model of primary anal canal adenocarcinoma with distinct cellular origins, etiologies, inflammatory microenvironments and mutational signatures: implications for personalised medicine. Br J Cancer. 2018;118(10): 1302-12.

23. Asgarova A, Asgarov K, Godet Y, Peixoto P, Nadaradjane A, Boyer-Guittaut $M$, et al. PD-L1 expression is regulated by both DNA methylation and NF-kB during EMT signaling in non-small cell lung carcinoma. Oncoimmunology. 2018;7(5):e1423170.

24. Ferrari de Andrade L, Tay RE, Pan D, Luoma AM, Ito $Y$, Badrinath $S$, et al. Antibody-mediated inhibition of MICA and MICB shedding promotes NK cell-driven tumor immunity. Science (New York, NY). 2018;359(6383): 1537-42.

25. Lu Y, Li S, Ma L, Li Y, Zhang X, Peng Q, et al. Type conversion of secretomes in a 3D TAM2 and HCC cell co-culture system and functional importance of CXCL2 in HCC. Sci Rep. 2016;6:24558.

26. Dabitao D, Hedrich CM, Wang F, Vacharathit V, Bream JH. Cell-specific requirements for STAT proteins and type I IFN receptor signaling discretely regulate IL-24 and IL-10 expression in NK cells and macrophages. J Immunol (Baltimore, Md : 1950). 2018;200(6):2154-64.

27. Fu Q, Sun $Y$, Tao $Y$, Piao $H$, Wang $X$, Luan $X$, et al. Involvement of the JAKSTAT pathway in collagen regulation of decidual NK cells. Am J Reprod Immunol (New York, NY: 1989). 2017;78(6).

28. Xu L, Chen X, Shen M, Yang DR, Fang L, Weng G, et al. Inhibition of IL-6JAK/Stat3 signaling in castration-resistant prostate cancer cells enhances the NK cell-mediated cytotoxicity via alteration of PD-L1/NKG2D ligand levels. Mol Oncol. 2018;12(3):269-86.

29. Park JY, Lee SH, Yoon SR, Park YJ, Jung H, Kim TD, et al. IL-15-induced IL-10 increases the cytolytic activity of human natural killer cells. Mol Cells. 2011; 32:265-72. 